\title{
Revisión histórica, dialectológica y sociolingüística del diminutivo en español
}

\author{
Historical, dialectological and sociolinguistic review of the diminutive in Spanish
}

Jingyuan $\mathrm{Hu}$

Sun Yat-sen University - China

Guangzhou, China

hujy73@mail.sysu.edu.cn

\section{RESUMEN}

El diminutivo en español es un proceso derivativo altamente productivo de formación de palabras, que cuenta con una bibliografía amplia en el campo de la lingüística. Con el objetivo de entender mejor la evolución y el estado de la cuestión de este elemento lingüístico, se ha llevado a cabo una descripción sincrónica del diminutivo en español incluyendo los aspectos de variantes de sufijos en torno a un eje temporal, geográfico y social; para ello se ha realizado una revisión histórica de la evolución de los sufijos diminutivos, de la elaboración de mapas destinados a trazar la preferencia de cada sufijación tanto en España como en América Latina, así como un enfoque sociolingüístico del diminutivo centrándose en edad, sexo y clase social. Finalmente, se ha observado en diversa bibliografía que existe un uso ligeramente a favor por parte del sexo femenino, por una clase socioeconómica más humilde y por su uso en el ambiente rural.

Palabras clave: diminutivo; sociolingüística; lengua española; sufijos; historia

\section{ABSTRACT}

The diminutive in Spanish is a highly productive derivative process of word formation with an extensive bibliography in the field of linguistics. In this article, in order to better understand the evolution and the status of the issue of this linguistic element, a synchronic description of the Spanish diminutive has been carried out. Said analysis here includes aspects of suffix variants as they relate to the temporal, geographical and social axis. Specifically, in this article a historical review of the evolution of diminutive suffixes has been carried out. We have also completed maps meant to trace suffixation preferences in Spain and in Latin America while employing a sociolinguistic approach to the diminutive focusing on age, sex and social class. Finally, it has been observed in various bibliographies that there is a slightly favorable use by the female sex, by a humbler socioeconomic class and by its use in the rural environment.

Keywords: diminutive; sociolinguistics; Spanish language; suffixes; history 


\section{Introducción}

En español, el uso del diminutivo es amplísimo y desempeña un papel muy relevante en las conversaciones cotidianas tanto en España como en América Latina. Los diminutivos, un tipo de sufijos apreciativos, también se denominan sufijos de afectación (Alonso, 1954), sufijos valorativos (Seco, 1968), sufijos evaluativos (Varela, 1990), sufijos potestativos o apelativos (Gómez Torrego, 2002 [1997]) o sufijos interpretativos (Hummel, 1997). Generalmente, su empleo proporciona la idea de que el tamaño es más reducido que el promedio. Sin embargo, además de este valor nocional, son llamados así porque en la actualidad presentan frecuentemente otras funciones semánticas o pragmáticas relacionadas con la emoción que otorguemos al objeto o a la persona referidos. Los sufijos diminutivos en español muestran un amplio repertorio (RAE, 2009, p. 647; Aguirre, 2013, p. 281): -ito/-ita (librito, malita), -illo/-illa (dinerillo, chiquilla), -ico/-ica (bolsico, ratica), -ín (ino)/-ina (pequeñín, tontina), -ete/-eta (guapete, amigueta) y otros menos populares y dialectales como -iño/-iña (besiño, pobriña) en Galicia, -uco/-uca (papeluco, tierruca) en Cantabria y algunos otros con propensión a mostrar un sentido despectivo como -ejo/-eja (animalejo, calleja) y -uelo/-uela (mozuelo, mujerzuela).

Es llamativo el hecho de que la función semántica y pragmática del diminutivo vaya más allá de la mera referencia al tamaño pequeño: se enfoca hacia una proyección sentimental que permite enriquecer las posibilidades expresivas y limar las asperezas comunicativas. Dicho de un modo más metafórico: de acuerdo con distintos contextos y la intención del hablante, usar bien el diminutivo en la comunicación es como poner azúcar a las palabras (afectividad), redondear las esquinas para que no causen roces o envolverlas en un manto de terciopelo (cortesía, atenuación), usar una daga con maldad (menosprecio) o con ingenio y astucia (ironía). En otras palabras, este elemento morfológico, que presenta una amplia variedad geográfica, es ubicuo en la interacción oral de los hablantes españoles e hispanoamericanos ya que refleja parte de su creatividad subjetiva.

En este estudio se pretende realizar una revisión histórica, dialectológica y sociolingüística del diminutivo desde una perspectiva holística con el fin de entender mejor la evolución y el estado de la cuestión de este elemento lingüístico, que puede ser el fundamento sólido para las futuras investigaciones.

\section{Método}

El trabajo de Garro et al., (2010, p. 186-187) estudia el léxico elegido para la reeducación de fonemas y orienta sobre la manera de investigar la variedad lingüística en base a tres ejes: las distintas variantes diacrónicas, la procedencia geográfica y el origen social. Es verdad que la diversidad de usos del diminutivo depende de la situación comunicativa temporal, social y geográfica en la que se utiliza y de acuerdo con el nivel de conciencia léxica de quien la aplica. Para ello resulta esclarecedor considerar su evolución semántica, acudir a su etimología desde el latín y considerar su amplia distribución desde el punto de vista dialectológico y sociocultural. En este trabajo seguiremos esta propuesta y estudiaremos las variedades del diminutivo según el eje temporal, el eje geográfico y el eje social.

Se describen de manera cronológica la evolución y las características del diminutivo citando las obras tanto clásicas como modernas. Asimismo, se escogen las muestras típicas de la formación de diminutivos principalmente de la Nueva Gramática de Lengua Española (RAE 2009). 


\section{Eje temporal}

En cuanto al eje temporal, se realiza un análisis de cada sufijo desde una perspectiva etimológica y en su desarrollo diacrónico tanto formal como semántico. En primer lugar, es conveniente reproducir dos párrafos de la Nueva Gramática de Lengua Española (RAE 2009, p. 630, en adelante NGLE):
El sufijo diminutivo más extendido en la actualidad en todo el mundo hispánico es -ito, -ita, aunque en algunas zonas del Caribe alterna con -ico, -ica. En la lengua medieval y en la clásica era predominante -illo, -illa (como lo fue también en latín el sufijo -ELLUS, -A, -UM). Con el tiempo, la forma -ito, -ita fue ganando a las demás en extensión; no obstante, como comentaremos más abajo, aquella forma mantuvo un alto grado de lexicalización. En el español europeo se usa -ico, -ica en zonas nororientales y meridionales de la península ibérica (en ciertos casos, en alternancia con-iquio).
El sufijo -ico, -ica ha reducido considerablemente su extensión, ya que en el español antiguo era de uso general en amplias zonas del centro y norte de España. En algunos países andinos, así como en una parte de las áreas centroamericana y caribeña, se ha desarrollado las variantes -itico, -itica, en la que -ico parece añadirse a -ito: ahoritica, cerquitica, chiquitico, hijitico, mismitico, nuevitico, etc.

Los sufijos -ico y -uelo eran comunes en la Edad Media, mientras que en el Siglo de Oro se incrementó el uso de -illo (Lang, 1990, p. 140). La presencia dominante de -ito se produce en la Época Moderna y su uso se ve incrementado por su adopción en Hispanoamérica. En concreto, la evolución histórica de los sufijos fue muy variada. La realización de la siguiente tabla 1 muestra su evolución según Náñez Fernández (2006 [1973]) y Pharies (2002):

Tabla 1. Evolución temporal de los sufijos diminutivos

\begin{tabular}{|c|c|}
\hline $\begin{array}{c}\text { Sufijos diminu- } \\
\text { tivos }\end{array}$ & Evolución en el tiempo \\
\hline$-i t o,-i t a$ & $\begin{array}{r}\text { Aparece en lexicalizaciones hasta que a finales del XV irrumpe en la literatura, plena- } \\
\text { mente productivo desde el siglo XV hasta hoy en día }\end{array}$ \\
\hline$-i c o,-i c a$ & Aparece en la segunda mitad del siglo XV y la segunda mitad del XVII \\
\hline$-i l l o,-i l l a$ & Aparece en el siglo X y se generaliza en los siglos XIV y XV; en el siglo XVI es superado \\
& por -ito \\
\hline- ete, -eta & Aparece hasta el siglo XVI \\
\hline- ín (ino), -ina & Apdad Media \\
\hline$-u c o,-u c a$ & Aparece en el siglo XVI siglo XIII y muy abundante en el Siglo de Oro \\
\hline$-u e l o,-u e l a$ & Aparece en el siglo XII y a partir del siglo XV se amplían en los textos \\
\hline$-e j o,-e j a$ & \\
\hline
\end{tabular}

Fuente: Náñez Fernández, 2006 [1973] y Pharies, 2002

Podemos observar que hasta mediados del siglo XV solo tres sufijos diminutivos se usaban de modo habitual: -illo, -uelo y -ejo. A partir del siglo XV, dos nuevos sufijos, -ito, -ico, empezaron a competir con ellos y, a lo largo del tiempo, el sufijo -ito se convirtió en el marco diminutivo más usual y menos dialectal en castellano medieval, lo que supuso que otros sufijos, particularmente -illo, ha sufrido muchos desgastes en el valor afectivo, lo que favorece su capacidad para producir 
formas lexicalizadas. También hay que tener en cuenta la importancia de -ete en aragonés medieval, ya que era el sufijo más generalizado en esta región, tal y como explica Uritani (1985, p. 204).

Las bases del diminutivo poseen una clara procedencia latina. Según los textos presentados por González Ollé (1962), Horcajada (1987, p. 68-71) afirma lo siguiente:

Tras un período de coexistencia al lado de -ulus, -ellus acaba imponiéndose en toda la Romania. Es el origen del castellano -i(e)llo (...). Las acabadas en -o/-a añaden -i(e)llo/-a, las terminadas en -e,-ezi(e)llo. Las que en latín mantuvieron -olu( $m),-a(m), u(m)$ exigen -uelo(...). La oposición tallito/tallecito es consecuencia del mantenimiento en latín con dos variantes: -ellus, icellus: castellano: -i(e)llo, -eci(e)llo.

La tabla 2 que figura a continuación pretende reflejar de manera sucinta la etimología y la historia del repertorio de sufijos apreciativos según la hipótesis de Pharies (2002):

Tabla 2. Etimología de sufijos diminutivos

\begin{tabular}{|c|c|}
\hline Sufijos diminutivos & Etimología (se remonta al latín) \\
\hline -ito o -ita & -ittus \\
\hline$-i c o$ o $-i c a$ & $*_{-}$-iccus \\
\hline -illo o -illa & -ellus \\
\hline -ete o-eta & $\begin{array}{c}\text { catalano-aragonés -et, -eta } \\
\text { no latino -ittus }\end{array}$ \\
\hline -ín (ino) o -ina & -ìnus (-ìnus-a-um) o -ìna-ae \\
\hline -iño o iña & $\begin{array}{c}\text { gallego -iño o portugués -inho, } \\
\text { lat. -inus - } a \text {-um }\end{array}$ \\
\hline$-u c o$ o-uca & ${ }^{*}-\bar{u} c c u$ \\
\hline -uelo o -uela & -ólus \\
\hline -еjo o -eja & -iculus - $a-u m$ \\
\hline -ucho o-ucha & -us-culus \\
\hline
\end{tabular}

Fuente: Pharies, 2002

Ahora bien, merece la pena hacer referencia al sufijo -ín o -ina que no era originariamente un sufijo diminutivo en latín, sino que se empleaba para formar adjetivos. Según Uritani (1985, p. 204): "En la Edad Media aparece únicamente en los documentos del territorio asturiano o leonés, y en ellos resulta indudable su función diminutiva."

Adicionalmente, es interesante apuntar que -ico (con ortografía -iko o -eziko) es el único sufijo diminutivo que usan los sefardíes, constituyendo una marcada característica del judeoespañol, como por ejemplo en dediko (dedillo) y manezika (manita) (Šmid 2002, p. 121).

Con respecto a la evolución semántica, los gramáticos han intentado diferenciar valores para cada diminutivo. Gonzalo Correas (1954 [1625]) en su Arte grande de la lengua castellana describe los valores de cada sufijo de la siguiente manera: 
Los en -ito significan con amor y bien querer.

Los en -izo (sic por ico) no con tanta afición.

Los en -uelo, desprecio.

Los demás casos, desdén.

Los en -ino disminuyen en gran medida.

Desde el punto de vista semántico, Hasselrot (1957, p. 258) hacía hincapié en que -ito es el sufijo más vivo y prácticamente el único capaz de expresar a la vez disminución y afecto. Según Náñez Fernández (2006 [1973], p. 326): "Desde el siglo XVIII hasta hoy, -ito es el más importante de los sufijos diminutivos”. De acuerdo con Lang (1990, p.140): “-ito es el más usado y el que se combina en mayor medida con cualquier tipo de bases; del mismo modo, es el menos marcado peyorativamente". Pharies (2002, p.367) invoca la descripción de Rainer (1993, p.578-589): "Además de los usos diminutivo e hipocorístico, se usa para connotar, en los derivados basados en sustantivos, ironía (ya tiene sus añitos), modestia (tengo unos terrenitos) o desprecio (empleadito), mientras que en los derivados basados en adjetivos se usa para intensificar (pequeñito), despertar simpatía (pobrecito) o atenuar (gordito)".

Igualmente, Lázaro Mora (1981, p. 494) señala que -ito, -illo, -ete, -ico contienen un significado tanto nocional como afectivo; este último es predominante salvo si el contexto lo elimina. El sufijo -ito es el más neutro y el menos marcado dialectalmente. Añade que los sufijos -ete/-eta poseen un sentido de connotación despreciativa más próximo a -illo que a -ito e -ico, y también afirma que el sentido connotativo de -ico está más próximo a -ito, alejándolo de -ete/-eta que están más próximos a -illo, que suele poseer el valor desdeñoso e irónico. Se puede observar este matiz en usos como: "Yo - comentaré con mis amigos- estoy escribiendo un articulillo (incluso un articulejo) para restar énfasis e importancia a mi acción. Un articulito manifestaría, como articulillo, lo reducido del trabajo, pero mi adhesión a él sería totalmente positiva" (Lázaro Mora, 1981, p. 487).

En opinión de Lang (1990, p. 143), -ete se caracteriza por su bajo matiz afectivo, pero su valor despectivo es inferior al que contiene -uelo, que en ocasiones puede resultar jocoso y ligeramente desdeñoso por "influencia de la vocal anterior / $\mathrm{u} /$ a la que se suele asociar un efecto fonosimbólico negativo" (Lang, 1990, p. 138). Por este motivo a veces se ha denominado "diminutivo burlador" como en Lázaro Mora (1981, p. 496). Asimismo, Miranda (1994, p. 124) considera que la semántica de -uelo se aproxima a -ete en el caso de que el matiz despectivo englobe un contenido jocoso y afectivo. Para Bajo Pérez (1997, p. 50), -ete implica una connotación pícara o burlona que lo distingue del resto de la sufijación diminutiva. Martín García (2016, p. 422) afirma que -ete es menos peyorativo que -uelo al marcar ironía y complicidad (Iadroncete/ladronzuelo).

Por su parte, Lang (1990, p. 146-147) comenta que el sufijo -ico dispone de mayor idiosincrasia dialectal entre todos los diminutivos y su matiz semántico se aproxima más a -ito por "su valor estrictamente diminutivo y medianamente afectivo". Además, dicho investigador sostiene que los sufijos -ino (ín), -ina conllevan un "moderado valor peyorativo" y un carácter netamente dialectal, por lo que resultan poco productivos en comparación con -ito, -illo y -ete. A su vez, Fernández Ramírez (1986, p. 76) apunta que -ín(a) posee una intensión expresiva que, sobre todo, se resalta en los usos hipocorísticos o peyorativos. 
En cuanto a otro sufijo dialectal como -uco, Pharies (2002, p. 511) afirma que en Santander de Tudanca “-uco, -uca es el sufijo más frecuentemente empleado (al lado de -ín) para añadir un matiz diminutivo y afectivo". Por otro lado, Bajo Pérez (1997, p. 60) indica que -ejo, -eja a veces puede señalar cierto afecto teñido de humor, aparte de ser sufijo despectivo, mientras que Pharies (2002, p. 511) comenta que el valor semántico actual de -ejo, -eja es diminutivo y peyorativo.

En la tesis doctoral de Espresati (2015, p. 298) se intenta sistematizar la magnitud escalar del valor de diferentes sufijos diminutivos exponiendo la siguiente gradación:

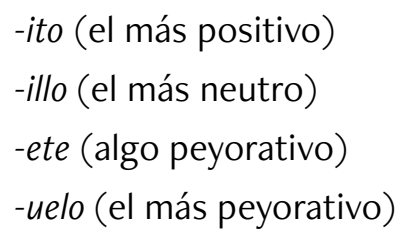

Por lo que atañe al restante de sufijos apreciativos tales como -ín, -iño, -uco, Espresati (2015, p. 298) advierte de que "no existe un consenso suficiente en cuanto a sus valores, que se manifiestan de un modo demasiado entremezclado, lo que puede inducir a pensar que estos dependen demasiado de los corpus de estudio en que se localizan y de otras variantes tal vez sociolingüísticas".

No obstante, por lo que hemos ido exponiendo hasta ahora, nos parece acertada esta escala de gradación de diminutivos y hemos optado por ilustrarla con los sufijos representativos desde el punto de vista semántico. Como se muestra en el Gráfico 1, del sufijo -ito,-ico, -illo, -ete al sufijo -uelo, el grado afectivo se reduce del nivel 4 al o de forma gradual, mientras que la connotación peyorativa aumenta del nivel o al 4 de forma progresiva. Sin embargo, hay que poner de relieve que se trata simplemente de una ilustración aislada del contexto y las diferencias geográficas, y que se debe distinguir el matiz concreto de un sufijo una vez que se coloque en una situación conversacional dada.

Gráfico 1. Contraste afectivo-despectivo

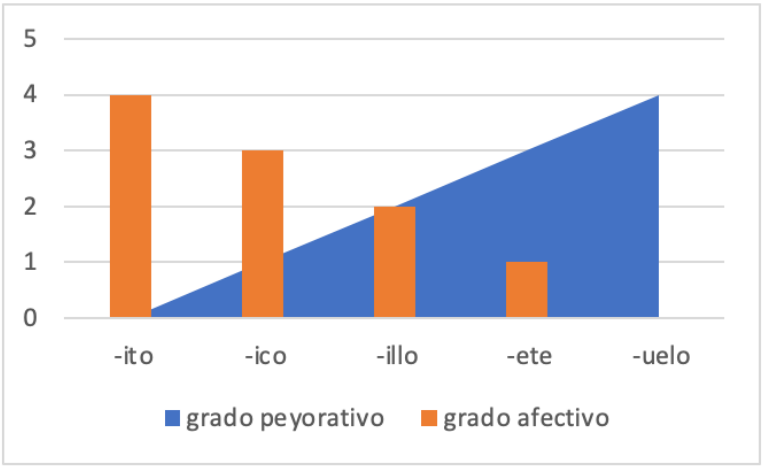

Fuente: Elaborado por la autora 


\section{Eje geográfico}

\section{1. Áreas españolas}

En lo que concierne a las variedades dialectales del diminutivo, Hasselrot (1957) es, sin duda, el primero que intentó ilustrar la distribución de distintos sufijos con mapas dialectales en territorio español y en Hispanoamérica. Según Hasselrot (1957, p. 264), a grandes rasgos, el panorama del diminutivo en España queda reflejado según la siguiente tabla, aunque se encuentra ausente la distribución de -ejo/-eja y -uelo/-uela:

Tabla 3. Área geográfica del diminutivo en España

\begin{tabular}{|c|c|}
\hline Tipo & Zona \\
\hline$-i \tilde{n}$ / - ín & Galicia, Asturias, León, Zamora, Salamanca y Extremadura \\
\hline$-u c o$ & Cantabria \\
\hline$-e t(e)$ & Cataluña, Valencia \\
\hline$-i c o$ & Navarra, Aragón, Cuenca, Albacete, mitad oriental de Andalucía, Murcia \\
\hline$-i l l o$ & Mitad occidental de Andalucía \\
\hline$-i t o$ & Mitad oriental de Castilla León, Madrid y Castilla La Mancha (excepto \\
& Cuenca y Albacete) \\
\hline
\end{tabular}

Fuente: Adaptado de Hasselrot, 1957

Posteriormente, los investigadores Urrutia y Álvarez (1988, p. 111-112) también elaboraron un mapa dialectal para presentar el uso del diminutivo en el territorio español: "En el centro predomina -ito. En Aragón -ico, también en Murcia y Granada, que, además, toma la variante -iquio. Por añadidura, podemos apreciar la presencia de -ico en una franja oriental de la península. En Andalucía hay -ito e -illo, con mayor abundancia de -illo que en Castilla”. Por otra parte, observamos que en Galicia predomina el sufijo -iño por influencia del gallego (Rojo, 2004, p. 1094), el sufijo -ín aparece en el Principado de Asturias y -uco es típico en Cantabria. No obstante, hay que destacar que la presencia de -cho en la zona del País Vasco e -iyo en Andalucía no parece realmente representativa. Además, en el mapa no se reflejan sufijos como -ete, -uelo y -ejo, por lo que nos resulta un mapa poco exhaustivo. Según Bajo Pérez (1997, p. 47-52), -ete figura en Cataluña, Valencia, Aragón, La Mancha y Murcia, la terminación - uelo tiene presencia en Murcia, La Mancha oriental, sur de Aragón y Andalucía oriental, y el sufijo -ejo es típico de Castilla-La Mancha.

Mapa 2. Área geográfica del diminutivo en España

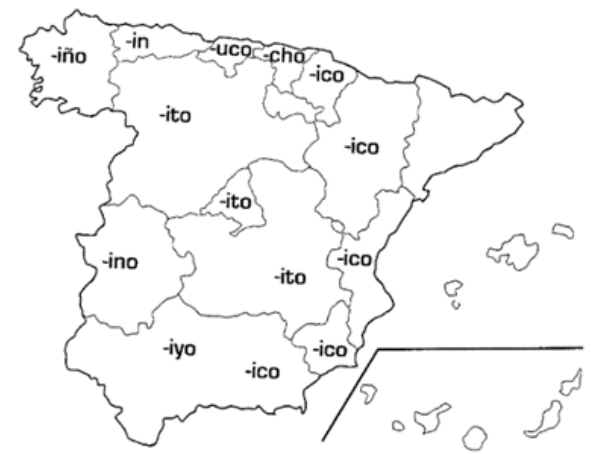

Fuente: Urrutia, C. H. y Álvarez, H. (1988, p. 111-112) 
Ahora bien, completando la propuesta de Bajo Pérez (1997, p. 47-52), la distribución geográfica de diminutivos se resume en la siguiente tabla:

Tabla 4. Área geográfica del diminutivo en España

\begin{tabular}{|c|c|}
\hline ito, ita & En todas partes, pero más habitual en Canarias y en América \\
\hline Ico, ica & $\begin{array}{c}\text { Valle del Ebro (Valencia, Murcia, Aragón, Navarra, Rioja...); Ilega hasta Granada; tam- } \\
\text { bién en parte de León, de Zamora y norte de Salamanca; habla rural de Murcia }\end{array}$ \\
\hline ín (-ino), ina & Desde Asturias hasta Extremadura; de Cantabria a Salamanca \\
\hline Iño, iña & Occidente de León y en toda Galicia \\
\hline et (ete), eta & Aragón, Mancha oriental, Andalucía oriental, Cataluña, Valencia y Murcia \\
\hline Illo, illa & En las hablas meridionales, sobre todo en el andaluz occidental \\
\hline Uco, uca & Asturias, Santander, norte de Burgos, de Palencia y León \\
\hline Uelo, uela & Murcia, Mancha oriental, sur de Aragón y Andalucía oriental. \\
\hline
\end{tabular}

Fuente: Bajo Pérez, 1997

Del mismo modo, en el periódico El País (31-01-2018), Pons Rodríguez publicó un artículo titulado "Una cosita que revela tu origen: el diminutivo" en el que señala que los diminutivos ofrecen pistas inequívocas de la procedencia de los hispanohablantes. Este texto expone que la primera pista es la mera elección del sufijo que se utiliza: el empleo de niñuco, tierruca (con el sufijo -uco/uca) implica una procedencia de Cantabria; la utilización de muchachino (con el sufijo -ín(o)/ina) muestra que se trata de un hablante peninsular occidental, tal como asturiano, leonés, extremeño o de la sierra de Huelva; la aplicación de besiños indica el origen de Galicia. Bonico (con -ico) revela los hablantes de las áreas andina, caribeña y centroamericana o, en el español de España, de la zona este (Aragón, La Mancha, zona oriental andaluza, o si es de Murcia incluso con-iquio...). Chiquillo (con -illo) muestra que probablemente es de Andalucía.

Hemos de subrayar que en la Tabla 4 aparece el uso predominante del sufijo -ito en Canarias. Más abajo mencionaremos este mismo fenómeno en América Latina, ya que es abundante en Cuba, Santo Domingo, Puerto Rico, Colombia y Río de la Plata Gonzáles Ollé (1962, p. 196). En palabras de Uritani (1985, p. 224), esto nos hace pensar en el importante papel que desempeñaron las Canarias como centro intermedio que unía la península y América.

En realidad, el comportamiento de los sufijos apreciativos es mucho más complejo y son bastante interesantes. Algunas de las particularidades que hay que considerar son las siguientes:

En lo relativo al sufijo -ico, Zamora Vicente (1985, p. 278-279) añadía que tenía vitalidad en Albacete, Murcia y Andalucía oriental; Náñez Fernández (2006 [1973], p. 33) delimita su uso a Aragón, Navarra, Murcia, Granada y la forma -iquio en Almería y Murcia como boniquio (bonico). Es interesante mencionar el trabajo del morfólogo zaragozano Serrano Dolader (2018), quien toma ejemplos de una conversación con su profesora Martín Zorraquino (zaragozana): "Pero Davidico, icórtate esas uñicas!”. En este texto, también se emplea “iatentica, por favor!” y “Mariantoñica”, con lo cual se puede intuir el uso de -ico en la zona de Aragón. Por otra parte, el estudio reciente de Pérez-Salazar (2020, p. 200) pone de manifiesto la pluralidad de los sufijos diminutivos en Navarra donde -ico es el más empleado, -illo mantiene su vigencia, -ito sigue en aumento y -ete ha reducido su presencia. 
En lo concerniente a -uco, Zamora Vicente (1985, p. 164) agregaba que, aparte del uso representativo que se hace en Santander, -uco posee un uso despectivo en Asturias (rapazuca), o en Babia y Laciana (mucheruca). Asimismo, Zamora Vicente (1985, p. 163) consideraba frecuente el uso de -ín/-ina en Asturias y el noroeste de León, sobre todo en La Cabrera, donde comparte la vitalidad con -ico, -ica: pequeñín, pequeñina; rapacín, rapacina.

Además, según la NGLE (RAE 2009, p. 631): “Se emplea -ín/-ina (librín, pequeñina) en la zona noroccidental de España. En la suroccidental se prefiere -ino/-ina (muchachino, poquino) [...]. Aunque -illo/-illa y-ete/-eta son generales en el español europeo, el primero destaca proporcionalmente en Andalucía, y el segundo en Aragón, Levante y Cataluña”. Alvar y Pottier (1983, p. 370-371) detallan que -ín se localiza en el noroeste del dominio leonés (Curueña, Armental el Bierzo) y en el resto -ino, extendiéndose por Extremadura y alcanzando a las zonas andaluzas limítrofes (Huelva, Sevilla, Córdoba). Su extensión por otras zonas es ocasional o responde a lexicalizaciones cumplidas en épocas antiguas (Miranda 1994, p. 124-125).

Por otra parte, debemos mencionar la distribución del sufijo -ejo, -eja, que "se aplica en la Mancha y en otras partes del área centromeridional de España” (NGLE, RAE 2009, p. 631), por ejemplo, familieja, tempanejo; además, Náñez Fernández (2006 [1973], p. 33) destaca la presencia de -ejo en Guadalajara. Sin embargo, cabe enfatizar que en muchos casos al añadir el sufijo -ejo/-eja en estas zonas no se añade un matiz peyorativo.

En definitiva, la distribución de los distintos sufijos del diminutivo es muy compleja. En el siguiente mapa presentamos una aproximación de las preferencias regionales de sufijos diminutivos que venimos revisando, pero debemos apuntar que figuran los predominantes, puesto que en cada territorio delimitado se pueden emplear otras terminaciones.

Mapa 3. Diminutivos en España.
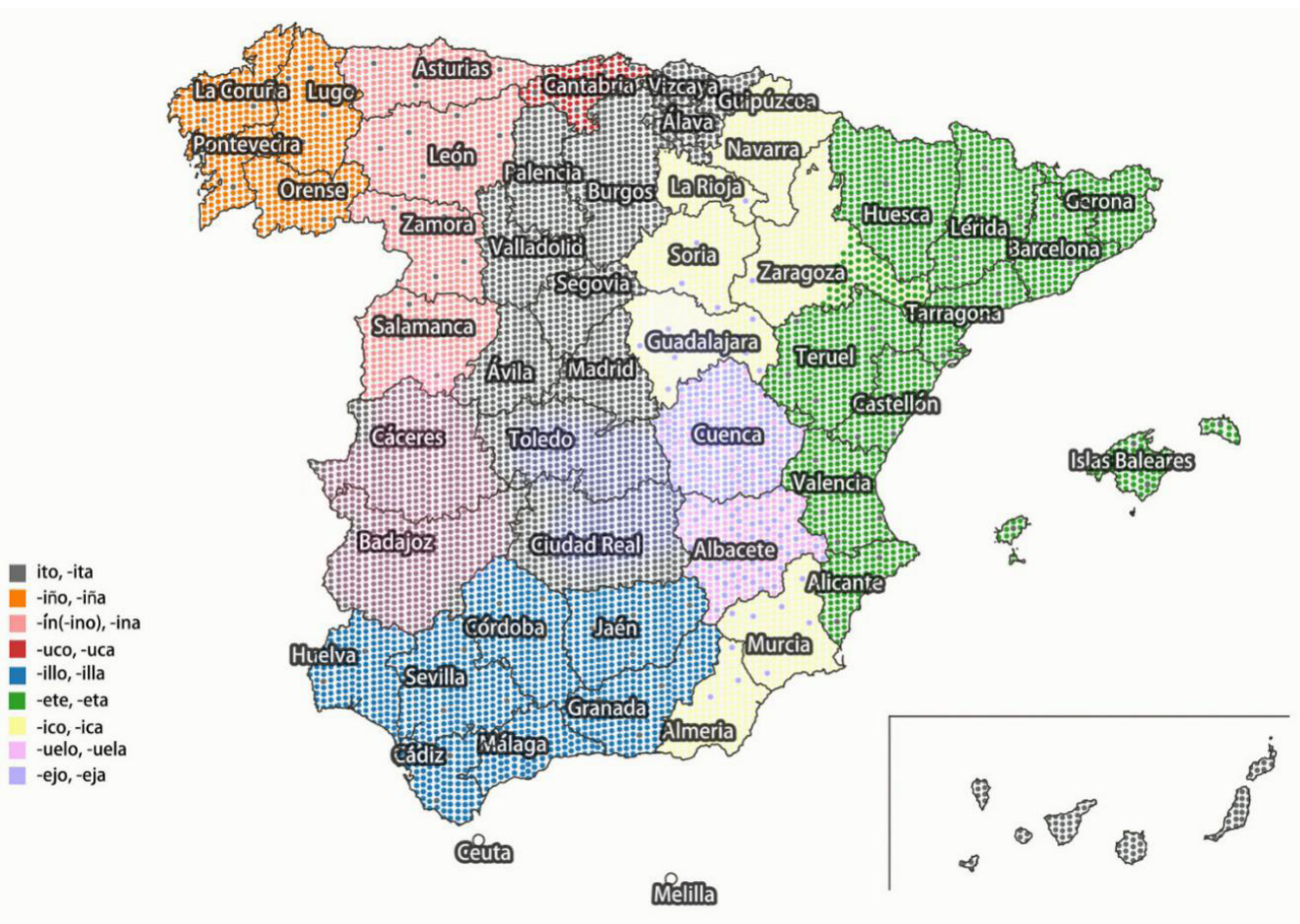

Fuente: Elaboración propia 


\section{2. Áreas latinoamericanas}

La otra propuesta elaborada por Hasselrot (1957, p. 269) acerca de la distribución de diminutivos en Hispanoamérica queda registrada como sigue:

Tabla 5. Área geográfica del diminutivo en América Latina

\begin{tabular}{|c|c|}
\hline -illo & Mitad occidental de Cuba \\
\hline -ito & México, mitad sur de Ecuador, Perú, Argentina, Chile, Paraguay y Uruguay \\
\hline -tico & Centroamérica (excepto la mitad occidental de Cuba), Venezuela, Colombia y la mitad norte de \\
& Ecuador \\
\hline
\end{tabular}

Fuente: Hasselrot, 1957

En cuanto a los tres sufijos mencionados, Hasselrot (1957) indica que -illo apenas aparece como sufijo diminutivo excepto en la mitad occidental de Cuba. Según Lipsky (1994, p. 304), en algunas regiones del sudeste de México, especialmente en Chiapas, sí es frecuente (pronunciado como [ío]), mientras Pharies (2002, p. 328) cita que -illo también es productivo como sufijo despectivo en México y Costa Rica. En opinión de Alba Ovalle (2001), en el español del Caribe se registran los sufijos -ín/-ina, -illo/a, -ito/a e -ico/a. Por otra parte, Lapesa (1980, p. 585) resalta que en América abundan palabras con -illo cuya noción no es la misma que la de los diminutivos primitivos, es decir, se orientan hacia la lexicalización. Por ejemplo: frutilla (fresa), tinterillo (abogado picapleitos). Además, existen muchos hipocorísticos con este sufijo: Arturillo, Martilla, Josecillo. A nuestro parecer, no se establecen con exactitud estas afirmaciones, ya que, aparte de las zonas mencionadas, es cierto que se registran chiquillo/chiquilla en Chile, Perú, Venezuela y Colombia según el Corpus de Referencia del Español Actual (CREA).

Ahora bien, a ambos lados del Atlántico el uso de -ito posee una gran vitalidad y coexiste con otros sufijos diminutivos preferentes. Tal como afirma Monge (1988, p. 130), -ito es el sufijo más productivo en todas las variedades del español. En la tabla 5 resulta obvio que el sufijo -ito se extiende en la mayor parte del continente en América Latina y constituye la aplicación por excelencia que se asocia a sus varias funciones pragmáticas. Asimismo, Regúnaga (2005, p. 253) destaca que en Santa Rosa (Argentina) también se utiliza -itito/a.

Es necesario apuntar al caso de -ico, que según datos de Náñez Fernández (2006 [1973], p. 33), tiene presencia en Colombia, Costa Rica, Santo Domingo y el departamento oriental de la isla de Cuba. La NGLE (RAE 2009, p. 630-631) indica: "En algunos países andinos, y en parte de las áreas centroamericana y caribeña, se ha desarrollado la variante -itico/-itica: ahoritica, cerquitica, chiquitico, hijitico, mismitico, nuevitico, pequeñitico, pueblitico, tiernitico, [...]. Piensan, en cambio, algunos autores que estos casos podrían constituir un proceso de disimilación de la segunda -t- (-itico por-itito)", siendo más frecuente su aparición "cuando la base léxica contiene una oclusiva sorda: cerquitica, poquitico, tiernitico". Lo mismo explica Pharies (2002, p. 307), el sufijo -ico se suele colocar detrás de las palabras que terminan en consonante <t> por una causa de eufonía, en los casos de tantico, gestico, cuantico, ratico. Asimismo, Pharies (2002, p. 308) determina el dominio de -tico en Cuba oriental, Costa Rica (lo cual explica el apodo nacional de ticos), la República Dominicana, Panamá, Venezuela y Colombia, pero no en Puerto Rico. Por su parte, Seco (1989, p. 208) destaca el uso de -ico (gatico, zapatico) en Colombia y Venezuela 
cuando precede una - $t$ - sustituyendo a -ito; Vaquero (1996, p. 27) considera el uso predominante de -ico en Centroamérica, Colombia y Cuba.

Por otra parte, algunos investigadores afirman que los sufijos diminutivos como -ete, -ín y -uco apenas se aplican como diminutivos en América Latina, pero sí existen muchas palabras lexicalizadas como clavete o valantín, también en los nombres propios como Bertuca, Martuca, Pedruco, Teresuca (Pharies, 2002, p. 511). Hay que hacer hincapié en el sufijo -ete: “En el español americano, es notable el uso de -etas como sufijo deverbativo epiceno para designar a los individuos que hacen una cosa de forma exagerada: acusetas, conversetas, escuchetas, Iloretas" (Pharies, 2002, p. 341).

En realidad, según Vaquero (1996, p. 28), Bajo Pérez (1997, p. 52) y la NGLE (RAE 2009, p. 631), en determinadas áreas latinoamericanas también aparecen sufijos propios como los siguientes:

-ejo, -eja: aparecen en las zonas del Río de la Plata, y del español andino.

-ingo, -inga: posee gran vitalidad en la parte oriental de Bolivia.

-acho, -acha: aparece en el andino peruano.

-ucho, -ucha: Argentina.

-oco, -oca: Chile.

Con el objetivo de ilustrar la distribución de diminutivos más típicos en Latinoamérica como -ito/$i t a,-i c o /-i c a,-i l l o /-i l l a$ y los autóctonos que se han mencionado arriba, presentamos el siguiente mapa de elaboración propia:

Mapa 4. Diminutivos y los sufijos autóctonos en América Latina.

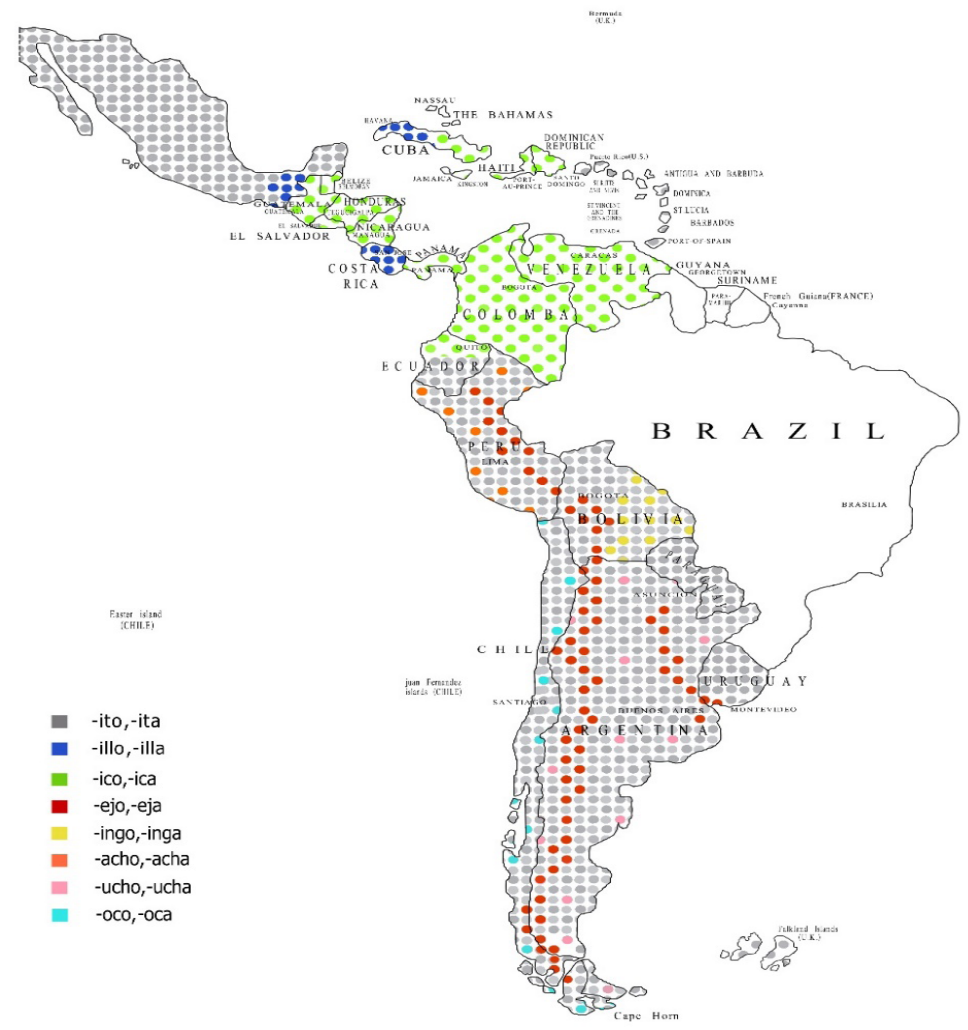

Fuente: Elaboración propia 
Es preciso poner de relieve que se trata de una aproximación de la distribución con respecto a preferencias regionales, ya que estos sufijos también pueden aparecer en otras regiones de manera esporádica.

Cabe destacar que, desde el punto de vista de Moreno Fernández (2010, p. 50), el diminutivo que se presenta en América refleja sus propios rasgos, a pesar de que a veces también se escuche en España, sobre todo en Andalucía y Canarias. Dicho autor atribuye este rasgo al plano gramatical, resaltando que el diminutivo de valor afectivo también se adjunta a adverbios o gerundios (corriendito). Por añadidura, también se emplea en numerales (primerito, ultimito), demostrativos (estito, esito, aquellito), posesivos (suyita, tuyito), interjecciones (ojito, hasta lueguito) etc., así como se construye mayoritariamente con el infijo -it- en vez de -ec-; esto es, se dice viejito, pancito y dulcito en lugar de viejecito, panecito y dulcecito.

El trabajo realizado por Jurafsky (1996) señala que el diminutivo semántico es polisémico y polifuncional, además, los sentidos y funciones que adopta pueden llegar a ser contradictorios a causa de las variedades regionales. Tal y como explica Rodríguez Muñoz (2012, p. 216), ahorita significa inmediatamente, en este preciso momento en México; en cambio, equivale a pronto, en un momentito en Cuba y República Dominicana.

De la misma manera, como pone de manifiesto la NGLE (RAE 2009, p. 636-637), es igualmente posible que el sufijo apreciativo aparezca en palabras lexicalizadas usadas solo en ciertas áreas. Por ejemplo:

En parte de México, así como en El Salvador y en otros países centroamericanos, se distinguen quesillo (diminutivo lexicalizado, en tanto en cuanto designa un tipo de queso diferente al queso ordinario) y quesito (diminutivo común); otro caso puede ser la voz lexicalizada de mantequilla, en parte del área rioplatense significa un tipo de bizcocho, mientras que, en el español europeo, en las Antillas y en el área andina implica un producto obtenido de la leche.

Asimismo, se documenta en NGLE (RAE 2009, p. 641): "Carlosito en Filipinas, y azucarita en algunas zonas del Caribe continental, Centroamérica y el área andina.” En algunas palabras como cuadrito, Jaimito, ruidito no se suele apreciar variación dialectal.

En opinión de Lang (1990, p. 140): "Cuanto más común sea la base, mayor variedad de diminutivo se le podrán adjuntar”. Esta idea es compartida por la NGLE (RAE 2009: 641) y así agrega que:

La existencia de tantos sufijos diminutivos sugiere que los hispanohablantes forman los derivados apreciativos de maneras distintas, dando preferencia a diversos factores gramaticales, entre los que está el mantenimiento de la consonante final y la supresión de una marca de palabra con la adición de un sufijo que haga expresar el género.

Es decir, la elección de la variante alomórfica suele estar individualmente condicionada y encierra relaciones estrechas con los factores regionales.

Por último, conviene señalar la cohesión lingüística a pesar de la variedad geográfica. Siguiendo a la NGLE (RAE 2009, p. 644): "Debido a que existe el gran número de intercambios lingüísticos entre España y América Latina que el cine y la televisión producen actualmente, así como la masiva inmigración, disminuyen en alguna medida las diferencias dialectales". 


\section{Eje social}

Con respecto al eje social, nos centramos en el valor sociolingüístico que encierra el diminutivo en las vertientes de sentido: niño/adulto, hombre/mujer, ciudad/rural, culto/popular y hablado/ escrito.

Según Dressler y Merlini (1994, p. 144), el componente principal de los diminutivos es el valor de informalidad ([non-serious $]$ ), cuya función consiste en "lowering one's responsibility towards the speech act being performed, or, more specifically, for lowering one's commitment to its illocutionary force". Por consiguiente, se favorece el uso del diminutivo, tal y como subraya Shivani (2009, p. 96), entre los enamorados y los niños, los padres y sus hijos, y también en los actos de habla dirigidos a los animales y las mascotas.

Del mismo modo, Fontanella (1962, p. 570) indica que el diminutivo ocupa un lugar muy relevante dentro de la lengua infantil, puesto que trata de una herramienta pertinente para transmitir contenidos afectivos y volitivos. En nuestra opinión, esto se debe a la aplicación de diminutivos de los padres con el fin de mostrar el cariño o, en ciertos casos, para amortiguar palabras incómodas o tabú como las referidas a los órganos sexuales (colita, pilila, pitilín, en el caso de los niños, y pochita, en el de las niñas, culete para el trasero, etc.), porque los niños suelen imitar y repetir las palabras de los padres; por otro lado, el empleo del diminutivo entre niños representa una actitud lúdica e imaginativa que se extiende hasta jugar con el lenguaje, por lo que Jurafsky (1996, p. 534) opina que el origen del diminutivo proviene del "niño".

Además, Fontanella (1962, p. 571) observa que los diminutivos son más utilizados entre las mujeres que entre los hombres en el habla bogotana debido a que "En el lenguaje femenino predominan la afectividad, la efusividad, la cortesía". En cuanto a la edad, dicha autora considera que los diminutivos se emplean con más frecuencia entre señoras de edad que entre chicas jóvenes. No obstante, en un reciente trabajo partido de un corpus de revistas mexicanas, Shivani (2009, p. 91) señalaba que el diminutivo es más usado en un público más joven, especialmente entre las mujeres o las chicas. Según la investigación de Hu (2020, p. 300) basada en un corpus audiovisual de 100 horas, se muestra una ligera predominancia en favor del sexo femenino y también un equilibrio entre diferentes generaciones.

Por otra parte, García Gallarín (2000, p. 383) pone de manifiesto que en la literatura medieval el diminutivo es una innovación lingüística elaborada por las mujeres, ya que se consideraba persona inferior al varón, física y mentalmente. Este discurso femenino se reflejaba en numerosas obras durante la Edad Media y el Siglo de Oro, aunque difícilmente se trasladó al habla masculina principalmente por la disparidad de la función sociocultural.

Espresati (2015, p. 465) concuerda en que el diminutivo es más propio de la lengua femenina, aún más, intenta arrojar luz sobre las relaciones entre los campos del sexo y del nivel sociocultural agregando que las intervenciones del diminutivo predominan en las mujeres del estrato sociocultural medio, más que las de los estratos bajos y altos; en lo relativo a los hombres, los niveles alto y medio se encontraban más igualados.

Más tarde, Vilchis y Sánchez (2015) llevan a cabo un estudio de los diminutivos enfocándose en el sexo y llegan a la misma conclusión: los hombres y las mujeres hacen un uso semántico-pragmático 
distinto del diminutivo. Esto es, las mujeres emplean este elemento con más frecuencia que los hombres con respecto al afecto y al desprecio. Asimismo, basado de la misma frecuencia de uso sustantivo, las mujeres aplican más diminutivos con adjetivos que los hombres, en cambio, estos emplean más diminutivos en los adverbios, por lo tanto, es posible caracterizar el género del hablante mediante el uso de este sufijo apreciativo.

No obstante, según el trabajo de Aleza Izquierdo (2016, p. 45), los jóvenes dominicanos no muestran una diferencia por sexo en el empleo del diminutivo. Asimismo, expone que son los hombres los que más atenúan la expresión utilizándolos y las mujeres son las que lo usan para expresar cariño y disminución de tamaño.

En resumen, según estos investigadores, la frecuencia del diminutivo en función del sexo depende del factor dialectológico y del corpus elegido, así como de los datos de los informantes.

En lo concerniente a la índole rural/urbana, en palabras de Alonso (1954, p. 212), la utilización del diminutivo se extiende más en zonas rurales que en las ciudades:

La abundancia del diminutivo es un rasgo regional, del habla de las regiones en cuanto que se opone a la general. Y como esta oposición es mayor en los campos que en las ciudades, es el diminutivo, sobre todo, un rasgo del habla rural [...].

Los ambientes rurales y dialectales que han creado y que cultivan estas maneras sociales suelen ser reacios a los modos de las relaciones interpersonales más disciplinadas de las ciudades o de las clases cultas, porque los juzgan más convencionales y más insinceros e inexpresivos que los suyos.

Pero, aunque los medios rurales son los más propicios para la creación y propagación de estas formas, no es admisible invertir los términos y decir que la abundancia de diminutivos sea un signo de popularismo, regionalismo o ruralismo.

Ahora bien, por lo que respecta al registro popular o vulgar de este elemento, no contamos con trabajos exhaustivos que ofrezcan una visión de conjunto en el mundo hispanohablante, sino que solo poseemos algunas aportaciones de variación sociopragmática regional. A través de los datos recopilados en Colombia, Fontanella (1962, p. 571) y Montes Giraldo (1972, p. 87) coinciden en la opinión de que el diminutivo posee un aspecto vulgar o popular y predomina entre las clases sociales bajas a causa de apocamiento y un sentimiento de inferioridad hacia las clases altas. Lo mismo opina Reynoso Noverón (2001, p. 134): “Los registros populares del español mexicano registran un número de diminutivos marcadamente superior a los registros cultos". Sin embargo, Zuluaga (1970, p. 46) opina que el diminutivo también puede aparecer en las clases elevadas en una determinada situación coloquial-familiar. En este plano, Ramírez Luengo (2006, p. 44) concuerda en la presencia mayoritaria del diminutivo en las clases populares y hace hincapié en que en Venezuela se inclinan casi exclusivamente por la forma -ito en vez de -illo o -uelo, apareciendo estos dos exclusivamente en las clases altas. La investigación de $\mathrm{Hu}$ (2020, p. 16) también muestra que el uso del diminutivo predomina ligeramente hacia los estratos socioeconómicos bajos.

Debido a la carga afectiva y popular del diminutivo, es muy frecuente asociarlo con textos coloquiales e informales en un entorno familiar o amistoso. Justamente la previsión de que el 
diminutivo se usa más en la lengua hablada que en la escrita se atestigua en el trabajo reciente de Criado de Diego, quien ha llevado a cabo un análisis comparativo basado en un corpus propio construido de textos escritos y orales, y del que consideramos conveniente citar su resultado:

Podemos concluir que la afirmación de que el diminutivo es más frecuente en la lengua oral que en la escrita debe matizarse. Es, seguramente, más habitual en textos dialógicos, orales y escritos, donde la interacción permite fácilmente la presencia de las funciones afines al diminutivo, como la afectividad, la cortesía, la intensificación o la ironía. Como es evidente, su naturaleza eminentemente afectiva o sentimental hará que tanto en la escritura como en la oralidad su uso sea prácticamente nulo en textos de carácter científico o tecnológico, y mucho más recurrente en textos ficcionales relativos al ocio y a la vida cotidiana (2016, p. 193).

\section{Conclusiones}

En este artículo se han examinado las características de diferentes variedades de sufijos diminutivos en torno a un eje temporal, geográfico y social. Históricamente, la mayoría de estos elementos apreciativos provienen etimológicamente del latín, aunque -ito posee un origen desconocido, y los sufijos -ico y -uco tienen una procedencia incierta. El sufijo -ín o -ina tampoco era originariamente un sufijo diminutivo en latín. Además, el inventario imperante del diminutivo sufre una evolución, -illo experimenta un desgaste a partir de la época medieval debido a la creación de numerosas voces lexicalizadas. Desde el siglo XV hasta hoy día, -ito se ha convertido en el sufijo predominante y es capaz de adjuntarse a variadas categorías gramaticales. En cambio, otros sufijos se enfrentan a más restricciones. Desde la perspectiva semántica, se considera que -ito, -ico,-illo, -ete y -uelo pueden mostrar respectivamente una gradación emotiva desde lo afectivo a lo peyorativo. Sin embargo, la función del diminutivo, tal como afectividad, tamaño pequeño, atenuación, cortesía, intensificación o menosprecio, es solo detectable contextualmente, porque la expresión está asociada a un propósito intencional y un mismo diminutivo puede ser peyorativo en este caso, pero afectivo en otro.

Desde el punto de vista geográfico, el repertorio de sufijos diminutivos en España es más amplio que el de Hispanoamérica. En este trabajo se han elaborado dos mapas con los diminutivos preferentes para cada región. Sin embargo, cabe poner de relieve la disminución de las diferencias dialectales por el frecuente intercambio lingüístico en nuestra era global, es decir, se pueden encontrar otras terminaciones fuera de regiones delimitadas.

Por otra parte, también se ha suscitado la revisión sociolingüística encaminada a determinar el sexo, la edad y la clase social del hablante. Se ha observado en diversa bibliografía que existe un uso ligeramente a favor por parte del sexo femenino, por una clase socioeconómica más humilde y por su uso en el ambiente rural. Asimismo, se ha constatado el rasgo informal, amistoso y coloquial que presenta el diminutivo. 


\section{REFERENCIAS}

Aguirre, C. (2013). Manual de Morfología del español. Castalia.

Alba, O. (2001, octubre 16-19). El español estándar desde la perspectiva dominicana. [Congreso] Actas del II Congreso Internacional de la Lengua Española. Valladolid, España.

Aleza Izquierdo, M. (2016). El diminutivo en el español de Santo Domingo. Círculo de Lingüística aplicada a la comunicación, 67, 3-56. https://doi.org/10.5209/CLAC.53476

Alonso, A. (1954). Noción, emoción, acción y fantasía en los diminutivos. Gredos.

Alvar, M., y Pottier, B. (1983). Morfología histórica del español. Gredos.

Bajo Pérez, E. (1997). La derivación nominal en español. Arco/Libros, S. L.

Criado De Diego, C. (2016). Variación y variedad del diminutivo en español y su aplicación a la enseñanza del español como lengua extranjera en Brasil [Tesis doctoral UNED] Madrid. E-espacio http://e-spacio. uned.es/fez/view/tesisuned:Filologia-Ccriado

Dressler, W. U., \& Merlini, L. (1994). Morphopragmatics: Diminutives and intensifiers in Italian, German, and other languages. Mouton de Gruyter.

Enguita Utrilla, J. M. (1984). Notas sobre los diminutivos en el espacio geográfico aragonés. Archivo de Filología Aragonesa, 34-35, 229-250.

Espresati, C. G. (2015). Problemas de morfopragmática del diminutivo en español [Tesis doctoral Universidad de Valladolid] Valladolid. UVaDoc https://uvadoc.uva.es/handle/10324/16500

Fernández Ramírez, S. (1986). La derivación nominal. Anejo XL del BRAE.

Fontanella De Weinberg, M. B. (1962). Algunas observaciones sobre el diminutivo en Bogotá. Boletín del Instituto Caro y Cuervo, XVII, 557-573.

García Gallarín, C. (2000). Los diminutivos en el discurso femenino (Edad Media y siglos de oro). Verba: Anuario galego de filoloxia, 27, 379-404.

Garro Andrada, V., Brinia, G., \& Jackeline N. M. (2010). Diminutivos y variedades lingüísticas: Análisis de su inclusión en la reeducación fonoaudiológica. Fundamentos en Humanidades, XI(21), 177-191. https:// www.redalyc.org/articulo.oa?id=18415426012

Gómez Torrego, L. (2002 [1997]). Gramática didáctica del español. S. M.

González Ollé, F. (1962). Los sufijos diminutivos en castellano medieval. CSIC.

Gonzalo, C. (1954[1625]). Arte grande de la lengua castellana. CSIC.

Hasselrot, B. (1957). Études sur la formation diminutive dans les langues romanes. Upsala: Universidad de Upsala.

Horcajada, B. (1987). Morfonología de los diminutivos formados sobre bases consonánticas monosilábicas. Revista de Filología Románica, (5), 55-72.

Hu, J. Y. (2020). La enseñanza del diminutivo en Español como Lengua Extranjera a sinohablantes a través de contenidos audiovisuales españoles e hispanoamericanos, [Tesis doctoral, Universidad Complutense de Madrid] Madrid. E-prints Complutense. https://eprints.ucm.es/id/eprint/63410/

Hummel, M. (1997). Para la lingüística de vuestro diminutivo: Los diminutivos como apreciativos. Anuario de Estudios Filológicos, XX, 191-210.

Jurafsky, D. (1996). Universal tendencies in the semantics of the diminutive. Language, 72(3), 534-578.

Lázaro Mora, F. (1981). Los derivados sustantivos con -ete, -eta. BRAE, (61), 481-496.

Lang, M. (1990). Formación de palabras en español. Cátedra.

Lapesa. R. (1980). Historia de la lengua española. Gredos. 
Lipski, J. M. (1994). El español de América. Cátedra.

Martín García, J. (2016). Aumentativos y diminutivos. En, J. Gutiérrez-Rexach. Enciclopedia de Lingüística Hispánica (416-422). Routledge.

Miranda, J. A. (1994). La formación de palabras en español. Colegio de España.

Monge Casao, F. (1988). Diminutivos: cuantificación, subjetividad, especialización. En J. Lüdke. (ed.) Energia und Ergon, III, Das sprachstheorische Denken Eugenio Coserius in der Discusion (2) (pp. 129-140). Túbingen.

Montes Giraldo, J. J. (1972). Funciones del diminutivo en español: ensayo de clasificación. Boletín del Instituto Caro y Cuervo, (27), 71-88.

Moreno Fernández, F. (2010). Las variedades de la lengua española y su enseñanza. Arco/Libros, S.L.

Náñez Fernandez, E. 2006 [1973]. El diminutivo. Historia y funciones en el español clásico y moderno. UAM Ediciones.

Pérez-Salazar, C. (2020). Entre trabajillos, mocetes, añitos y momenticos. Aproximación a la forma y significado del diminutivo en Navarra. Revista de Filología, 40, 181-203. https://www.ull.es/revistas/ index.php/filologia/article/view/997

Pharies, D. (2002). Diccionario etimológico de los sufijos españoles. Gredos.

Quintana, A. (2001). Concomitancias lingüísticas entre el aragonés y el ladino (judeoespañol). Archivo de filología aragonesa, 57-58, 163-192.

RAE. (2009). Nueva gramática de la lengua española. Espasa Libros.

Rainer, F. (1993). Spanische Wortbildungslehre. Max Niemeyer.

Ramírez Luengo, J. L. (2006). Una nota de sociolingüística histórica: el diminutivo en el español uruguayo del siglo XIX. Res Diachronicae, 5, 39-45.

Regúnaga, A. (2005). Morfología derivativa: consideraciones en torno al uso de diminutivos en la ciudad de Santa Rosa (La Pampa-Argentina). Anclajes, 9(9), 251-262. https://cerac.unlpam.edu.ar/index.php/ anclajes/article/view/306

Reynoso Noverón, J. (2001). Los diminutivos en el español. Un estudio dialectología comparada. (Tesis doctoral). Universidad Nacional Autónoma de México, México.

Rodríguez-Muñoz, F. J. (2012). ¿Neutralización, remodalización o subjetivación? A propósito de la combinación 'muy + adjetivo diminutivo' en español. Revista española de lingüística aplicada, 25, 211223.

Rojo, G. (2004). El español de Galicia. Ariel.

Seco, R. (1968). Manual de Gramática Española. Aguilar.

Seco, M. (1989). Gramática esencial del español: introducción al estudio de la lengua. Espasa-Calpe.

Serrano Dolader, D. (2018). Pero Davidico, icórtate esas uñicas! [Congreso] Jornada de Homenaje con motivo de su 70 aniversario, María Antonia Martín Zorraquino, Filología y Vida. Encuentro de investigadores, profesores y discípulos. Zaragoza, España.

Shivani. J. (2009). Uso y función de los diminutivos en revistas para jóvenes mexicanas. Chrestomathy: Annual Review of Undergraduate Research, School of Humanities and Social Sciences, School of Languages, Cultures, and World Affairs, 8, 77-98.

Šmid, K. (2002). Los problemas del estudio de la lengua sefardí. Verba hispánica 1(10), 113-124. https://doi. org/10.4312/vh.10.1.113-124

Uritani, A. B. y Uritani, N. (1985). Los diminutivos en los atlas lingüísticos españoles. LEA: Lingüística española actual, $7,203-236$. 
Urrutia, C. H., y Álvarez, H. (1988). Esquema de morfosintaxis histórica del español. Publicaciones de la Universidad de Deusto.

Vaquero De Ramírez, M. (1996). El español de América II. Morfosintaxis y léxico. Arco Libros.

Varela Ortega, S. (1990). Fundamentos de morfología. Gredos.

Vilchis Vargas, E., y Sánchez Velázquez O. C. (2015). Hombrecillos y mujercitas: estudio de los diminutivos para detección de género [Ponencia]. V seminario de Lingüística Forense en la UNAM, México.

Zamora Elizondo, H. (1945). Los diminutivos en Costa Rica. Thesaurus, 3, 540-546.

Zamora Vicente, A. (1985). Dialectología española. Gredos.

Zuluaga Ospina, A. (1970). La función del diminutivo en español. Boletín del Instituto Caro y Cuervo, 25, 2348.

\section{AUTORA}

Jingyuan Hu. Doctora en lengua española y literatura por la Universidad Complutense de Madrid. Profesora del Departamento de Lenguas Extranjeras de la Universidad Sun Yat-sen de Guangzhou, China. Su línea de investigación gira en torno a la derivación apreciativa, la morfología y la enseñanza del español a estudiantes chinos.

\section{Conflicto de intereses}

La autora informa que no existe conflicto de interés posible.

\section{Financiamiento}

No existió asistencia financiera de partes externas al presente artículo.

\section{Agradecimientos}

\section{$\mathrm{N} / \mathrm{A}$}

\section{Nota}

El artículo es un producto de mi tesis doctoral: La enseñanza del diminutivo en Español como Lengua Extranjera a hispanohablantes a través de contenidos audiovisuales españoles e hispanoamericanos [The Teaching of the Diminutive in Spanish as a Foreign Language to Native Chinese Speakers through Spanish and Spanish-American Audiovisual Content], Universidad Complutense de Madrid, 2020 